\title{
EPR Discovery of a New Pressure-Induced Low-Spin Phase in $(2 \mathrm{Me}-5 \mathrm{Et}-\mathrm{PyH})\left[\mathrm{Fe}(\mathrm{Th}-5 \mathrm{Cl}-\mathrm{Sa})_{2}\right]$
}

\author{
A. Krupska ${ }^{a}$, M.A. Augustyniak-Jabeokow ${ }^{a}$, \\ Yu.V. YABlOKOV ${ }^{a}$, V.V. Zelentsov ${ }^{b}$, V.A. UlanOV ${ }^{c}$ \\ AND J. MROZIŃSKI ${ }^{d}$ \\ ${ }^{a}$ Institute of Molecular Physics, Polish Academy of Sciences \\ M. Smoluchowskiego 17, 60-179 Poznań, Poland \\ ${ }^{b}$ Moscow Physical Technical Institute, 141700 Dolgoprudnii, Russia \\ ${ }^{c}$ Kazan Physical Technical Institute RAN \\ Sibirskii Trakt 10/7, 420029 Kazan, Russia \\ ${ }^{d}$ Faculty of Chemistry, University of Wrocław \\ F. Joliot-Curie 14, 50-383 Wrocław, Poland
}

(Received April 6, 2006)

Electron paramagnetic resonance studies of the high-spin (HS) $\leftrightarrow$ low-spin (LS) transition in 2-methyl-5-ethyl-pyridine-5-chloro-salicylalthiosemicarbazonatoferrate(III) performed under hydrostatic pressure up to $500 \mathrm{MPa}$ in a temperature range of $80-310 \mathrm{~K}$ have revealed two modifications of the low spin complexes: low-pressure (LS1) and high-pressure (LS2) ones. Under atmospheric pressure LS1 appears on cooling and disappears on heating at $220 \mathrm{~K}$. The hydrostatic pressure shifts the transition to higher temperatures. Below $275 \mathrm{~K}$ an increase in pressure to $410 \mathrm{MPa}$ results in abrupt changes in the $g$-factor and width $\Delta B$ of the EPR line indicating a transition to a new phase. The pressure-induced transition LS1 $\leftrightarrow$ LS2 is almost independent of $T$ up to $275 \mathrm{~K}$, where at a pressure of $420 \mathrm{MPa}$ a triple point is observed. The LS1 $\leftrightarrow$ LS2 and HS $\leftrightarrow$ LS2 (at $T>260 \mathrm{~K}$ ) transitions occur with a large hysteresis of about $95 \mathrm{MPa}$. The process of the spin transition has been shown to begin with the formation of domains of LS complexes in the matrix of HS ones. The response of the domains to external factors has been studied.

PACS numbers: 76.30.Fc, 75.30.Wx

\section{Introduction}

A lot of the $3 d^{4}-3 d^{7}$ metal complexes are known to undergo reversible transitions between high-spin (HS) and low-spin (LS) states induced by changes in 
temperature, pressure or by light irradiation [1-5]. The necessary condition of the spin transition is the vicinity of the energy terms of different multiplicity. The spin crossover phenomenon in $\mathrm{Fe}(\mathrm{III})$ consists in intra-ionic transfer of electrons between $\left(t_{2 \mathrm{~g}}\right)^{3}\left(e_{\mathrm{g}}\right)^{2}$ and $\left(t_{2 \mathrm{~g}}\right)^{5}\left(e_{\mathrm{g}}\right)^{0}$ configurations accompanied by a change in the spin state, $S=5 / 2 \leftrightarrow S=1 / 2$. There are different types of spin transition behaviour, with a gradual transition and an abrupt one as the most typical cases. The spin crossover has been extensively studied employing various experimental techniques (magnetic susceptibility, X-ray diffraction, Mössbauer spectroscopy, EPR, etc.) $[2,3]$. In iron(III) complexes the HS $\leftrightarrow$ LS transition appreciably changes the EPR spectrum [6] and EPR allows practically direct confirmation of the presence of the spin transition phenomenon [7-12]. EPR has been used for analysis of the nature of the ground state complex in the low-spin state $[8,9]$, to determine the rate at which the complex interconverts between HS and LS states $[10,11]$, to check the effect of the state of substance on the spin crossover $[12,13]$ and to study the arrangement of magnetic complexes in the new state and interactions between complexes [14-16]. Recently, direct observation of the low-spin domains formation in the process of spin transition $[15,16]$ has been made for some representatives of the vast class of the $\mathrm{Fe}(\mathrm{III})$ thiosemicarbazonates of the general formula $\mathrm{M}^{+}\left[\mathrm{Fe}(\mathrm{Th}-\mathrm{R}-\mathrm{Sa})_{2}\right]^{-}[3,5]$, where $\mathrm{M}=\mathrm{H}$, Li, Na, Cs or 2Me-5Et-PyH and $\mathrm{R}=\mathrm{Cl}, \mathrm{Br}$ and others.

One of the important features of a spin transition is its hysteresis. The compounds with thermal or pressure hysteresis of the spin transition are the most attractive as this phenomenon indicates that the complexes are able to exist in two different electronic states at the same temperature or pressure (bistability phenomenon potentially important for molecular electronics) [17, 18].

The study of spin crossover under high pressure offers a particular possibility to get insight into the nature of spin transition $[16,18,19]$. Because LS iron complexes are smaller than HS ones, the pressure favours the lesser LS complexes. Thus, the LS state gets stabilised under pressure and an increase in the transition temperature with increasing pressure is observed.

In this paper we present the EPR study of spin transitions in polycrystalline 2-methyl-5-ethyl-pyridine-5-chloro-salicylalthiosemicarbazonatoferrate(III), $(2 \mathrm{Me}-5 \mathrm{Et}-\mathrm{PyH})\left[\mathrm{Fe}(\mathrm{Th}-5 \mathrm{Cl}-\mathrm{Sa})_{2}\right]$, under ambient and hydrostatic pressure up to $550 \mathrm{MPa}$ in a wide temperature range (magnetic susceptibility and Mössbauer spectra of this compound have been measured previously [3]). Changes in the $g$-value, width, and intensity of the EPR line of complexes in the LS state are analysed to get information on the interaction between the complexes in the domains and on their interaction with the surrounding. On the basis of the EPR data a spin transition diagram confirming the discovery of a new type of bistability is proposed. 


\section{The structure of the Fe(III) complex}

The crystal structure of $(2 \mathrm{Me}-5 \mathrm{Et}-\mathrm{PyH})\left[\mathrm{Fe}(\mathrm{Th}-5 \mathrm{Cl}-\mathrm{Sa})_{2}\right]$ is not resolved. But, the structure of the complex anion is known for a related $\mathrm{NH}_{4}\left[\mathrm{Fe}(\mathrm{Th}-5 \mathrm{Cl}-\mathrm{Sa})_{2}\right]$, which crystallises in the rhombic Pnca space group with $Z=4$ and for some other compounds of this family $[3,20]$. The structure of $\mathrm{Fe}(\mathrm{III})$ complex is similar in all $\mathrm{M}\left[\mathrm{Fe}(\mathrm{Th}-\mathrm{R}-\mathrm{Sa})_{2}\right]$ compounds. The $\mathrm{Fe}(\mathrm{III})$ ion is coordinated by two R-salicylalthiosemicarbazone ONS-ligands (Fig. 1) in two

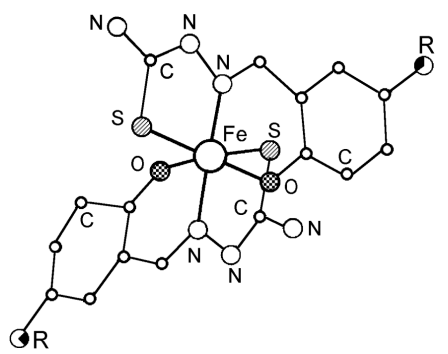

Fig. 1. The molecular structure of the $\mathrm{Fe}^{3+}$ complex coordinated by two 5-chlorosalicylaltiosemicarbazone ONS-ligands.

mutually perpendicular planes with $\mathrm{O}$ and $\mathrm{S}$ atoms in cis and $\mathrm{N}$ atoms in trans positions. The $\mathrm{Fe}\left[\mathrm{O}_{2} \mathrm{~N}_{2} \mathrm{~S}_{2}\right]$ octahedron is larger in the HS state than in the LS one and strongly distorted. The Fe-N and Fe-S distances are shorter in the LS state by about $0.2 \AA$, the $\mathrm{L}-\mathrm{Fe}-\mathrm{L}$ angles are also changed $[3,12]$. The crystal structure is built of cations $\mathrm{M}^{+}$and complex anions $\left[\mathrm{Fe}(\mathrm{Th}-\mathrm{R}-\mathrm{Sa})_{2}\right]$ linked by hydrogen bonds.

\section{Experimental}

The powder sample of $(2 \mathrm{Me}-5 \mathrm{Et}-\mathrm{PyH})\left[\mathrm{Fe}(\mathrm{Th}-5 \mathrm{Cl}-\mathrm{Sa})_{2}\right]$ was prepared by the method described in [21]. To the boiling water solution of $(2 \mathrm{Me}-5 \mathrm{Et}-\mathrm{PyH})$-thiosemicarbazonate a stoichiometric amount of water solution of $\mathrm{Fe}\left(\mathrm{NO}_{3}\right)_{3} \cdot 9 \mathrm{H}_{2} \mathrm{O}$ was added. The mixture was stirred and left to cool. The black crystalline precipitation was filtered off and washed with water, alcohol, and ether.

EPR spectra were recorded under atmospheric pressure on an X-band RADIOPAN SE/X spectrometer in temperatures 40-300 K. Next the same sample was studied by means of special high-pressure EPR equipment described in [22]. The measurements were carried out at different temperatures down to $150 \mathrm{~K}$ kept constant while changing the pressure up and down between the atmospheric pressure and $550 \mathrm{MPa}$. Thus, the effect of pressure hysteresis was possible to observe. The temperature changes in the EPR spectra at some constant pressures were recorded too. The temperature measurements under the ambient pressure were also made at a Q-band Varian E-12 spectrometer. 


\section{Analysis of EPR spectra}

The spin transition in iron(III) between the HS and LS states is responsible for strong temperature and pressure dependences of the shape of the EPR spectrum. The signal of the powder sample of HS iron(III) complexes $\left(t_{2 \mathrm{~g}}^{3} e_{\mathrm{g}}^{2}\right.$, $S=5 / 2$ ) in the compound studied at room temperature is a broad single line with $\Delta B=81.5 \mathrm{mT}$ and the $g$-factor value of $g=2.005$. The shape of this line can be approximated to sufficient accuracy with a Lorentzian line. The corundum resonator, used in high-pressure EPR measurements, contains some paramagnetic impurities [22]. Their cumulative EPR signal can be also approximated by a Lorentzian curve with different parameters. The EPR signal of the complexes in LS state $\left(t_{2 \mathrm{~g}}^{5} e_{\mathrm{g}}^{0}, S=1 / 2\right)$, which appears with decreasing temperature or increasing hydrostatic pressure, is totally different: it has much smaller $\Delta B$ and an anisotropic $g$-factor (Fig. 2). We are interested in the behaviour of the LS signal
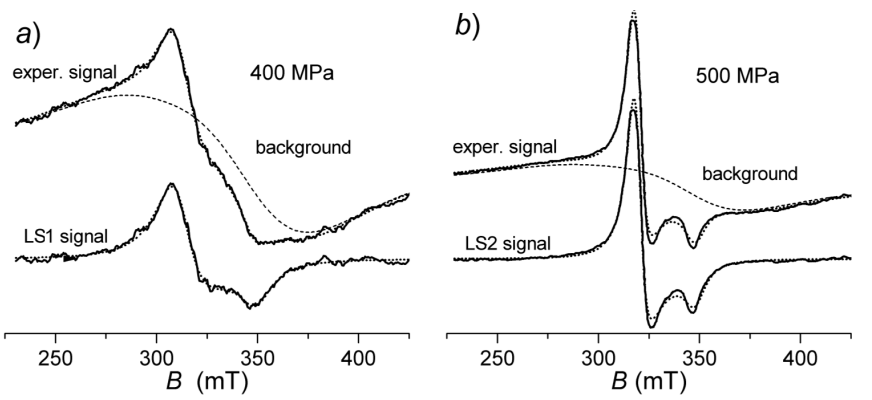

Fig. 2. Analysis of the X-band EPR spectra recorded at $260 \mathrm{~K}$ under pressure $400 \mathrm{MPa}$ (a) and $500 \mathrm{MPa}$ (b). The experimental spectra are given by solid upper line. The lower solid line shows the LS spectrum obtained by subtraction of the background (dashed line) from the experimental curve. The dotted lines represent the best fitting data to the experimental spectrum. The fitting parameters are: $g_{\|}=1.983, g_{\perp}=2.216, \Delta B_{\|}=10.9 \mathrm{mT}, \Delta B_{\perp}=15.3 \mathrm{mT}$ for $500 \mathrm{MPa}$ and $g_{\|}=1.989, g_{\perp}=2.166, \Delta B=7.6 \mathrm{mT}, \Delta B_{\perp}=6.5 \mathrm{mT}$ for $500 \mathrm{MPa}$.

and it is necessary to extract it from the spectrum observed. It is achieved by the numerical fitting procedure. The values of the $g$-tensor components, linewidths $\Delta B_{i}$ and integral intensity of the EPR signal from LS complexes as well as the data of background signals are obtained. The $\mathrm{X}$-band spectra suggest an axial symmetry of the LS centres. Although the $Q$-band measurements have shown some difference between $g_{x}$ and $g_{y}$ components of the $g$-tensor, it was so small $\left(\Delta g_{x, y}=0.03\right.$, see below) that we can restrict our considerations to the axial symmetry approximation in fitting the $\mathrm{X}$-band spectra.

As an example, the subtraction procedure of the background signals from the experimental one is shown in Fig. 2, at $400 \mathrm{MPa}$ and $500 \mathrm{MPa}$. Such a fitting procedure was carried out for all spectra recorded (for temperature measurements 


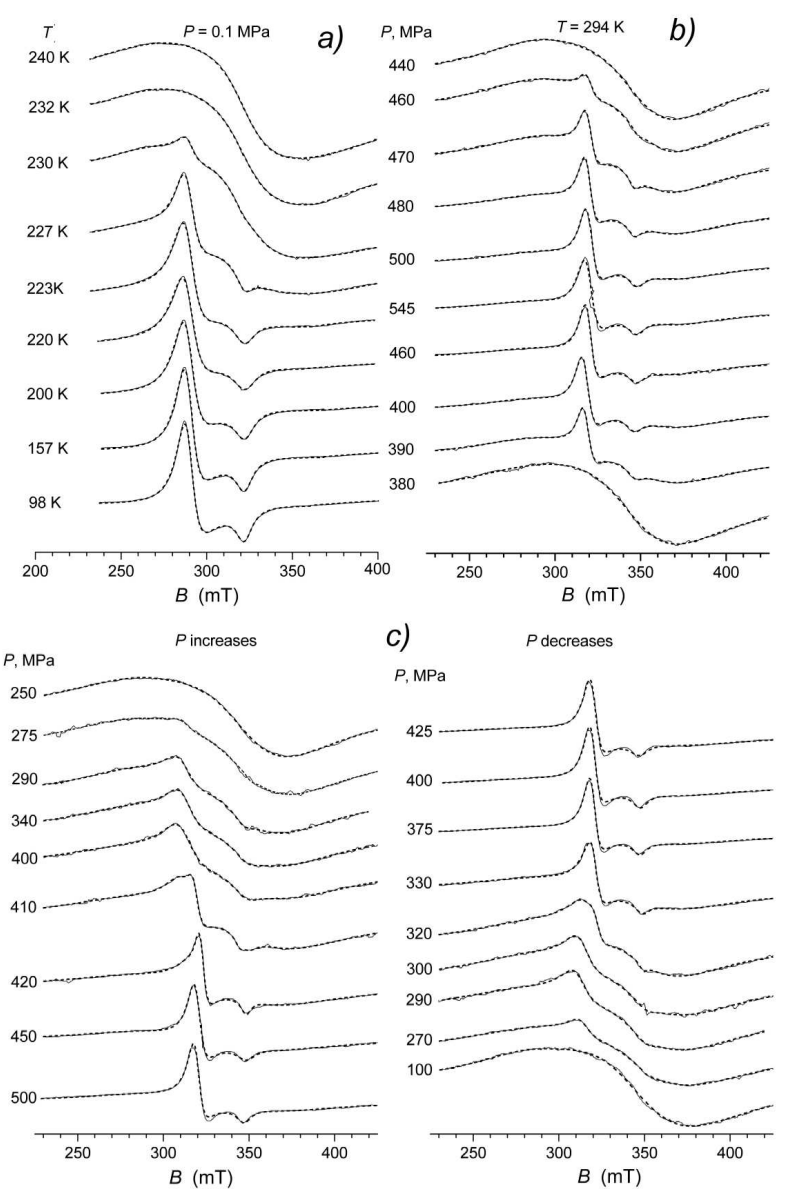

Fig. 3. Evolution of the experimental EPR spectrum of (2Me-5Et-PyH) $\left[\mathrm{Fe}(\mathrm{Th}-5 \mathrm{Cl}-\mathrm{Sa})_{2}\right]$ with the fitted one (dotted line) for three cases: (a) the temperature measurements at atmospheric pressure, (b) under various pressures at room temperature, and (c) at a constant temperature of $260 \mathrm{~K}$ during increasing pressure (left column) and decreasing pressure (right column).

at atmospheric pressure (a), under various pressures at room temperature (b) and at a constant temperature $260 \mathrm{~K}$ ), some of which are shown in Fig. 3. In the experiment under the ambient pressure we applied also direct subtraction of the high temperature signal from the spectra observed at lower temperatures. The results were in close agreement.

\section{Results}

\subsection{Parameters of the low-spin spectrum}

On cooling under atmospheric pressure the EPR signal assigned to the LS iron(III) complexes appears at about $230 \mathrm{~K}$. The integral intensity of this signal 
was estimated to be about $0.7 \%$ of that of the total signal (LS + HS). The intensity of the LS signal gradually increases with decreasing temperature, essentially exceeding the growth resulting from the changes in the population of the states split by magnetic field and described by the Boltzmann law (Fig. 3a and 4). On

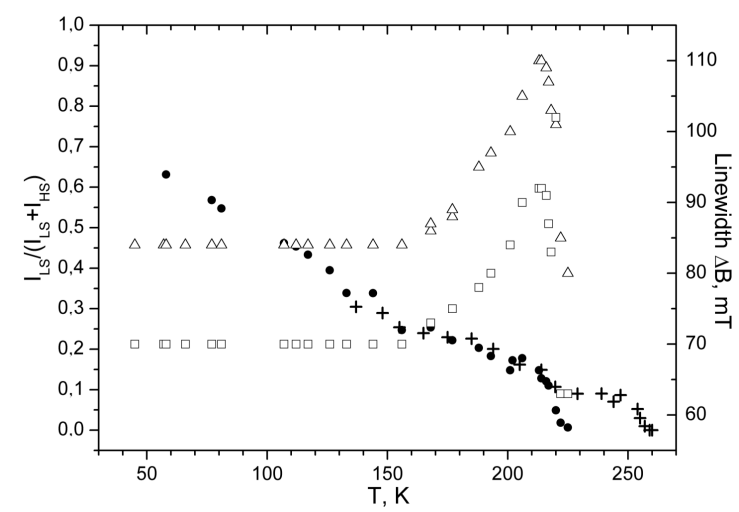

Fig. 4. The temperature dependences of $\Delta B_{\|}(\square), \Delta B_{\perp}(\Delta)$ values obtained from the fitting procedure and of the rate of integral intensities $I_{\mathrm{LS}} /\left(I_{\mathrm{LS}}+I_{\mathrm{HS}}\right)$ at ambient pressure $(\bullet)$ and at a pressure of $300 \mathrm{MPa}(+)$.

heating, the changes in the signal intensity are reverse; the hysteresis is not observed within the limit of experimental errors. The contribution of the LS phase seen by EPR increases relatively fast after the signal appearance, then it gradually grows with decreasing temperature. At $50 \mathrm{~K}$ the relative content of the LS complexes reaches $65 \%$. Figure 4 presents the temperature dependence of the LS signal integral intensity and that of parallel and perpendicular linewidths derived from the fitting procedure. As follows from Fig. 4, the transition to LS phase is not complete at $50 \mathrm{~K}$. This corresponds to the result obtained from the Mössbauer spectra analysis [3]. The values of the linewidths are different in $g_{\|}$and $g_{\perp}$ directions. The behaviour of the signal linewidth with temperature is not usual. In the beginning of the transition to the LS state the linewidth rises relatively quickly and then gradually decreases. Below $150 \mathrm{~K}$, the individual linewidths can be treated as independent of temperature. The $g_{i}$-values of LS spectrum are also independent of temperature. $Q$-band measurements give $g_{x}=2.228, g_{y}=2.203$, and $g_{z}=1.989$ (Fig. 5). The $g_{\|}=1.988, g_{\perp}=2.208$ values obtained by fitting to the $\mathrm{X}$-band spectra quite well agree with $g_{z}$ and $\left(g_{x}+g_{y}\right) / 2 Q$-band values.

The high-spin $\leftrightarrow$ low-spin transition can be also induced by hydrostatic pressure. However, the character of this transition and the EPR features of the LS complexes are essentially different. Figure $3 \mathrm{~b}$ shows the pressure induced spectrum evolution at $294 \mathrm{~K}$, which begins under about $460 \mathrm{MPa}$ and corresponds to the transition to the low-spin state. The value of $g_{\perp}=2.173(7)$ for LS high-pressure phase is smaller than that observed for the temperature induced LS centres under 


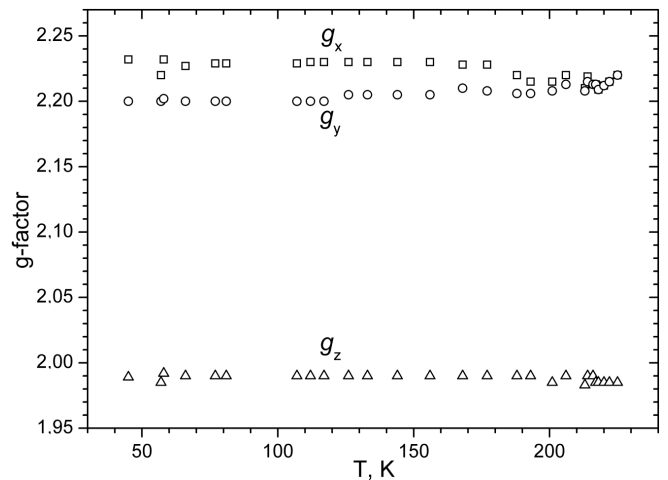

Fig. 5. The temperature dependences of $g_{x}, g_{y}$, and $g_{z}$-values obtained from the fitting procedure of $Q$-band EPR spectra.

atmospheric pressure, whereas $g_{\|}=1.997(5)$ is within the accuracy limits of the fitting procedure. Also, the mean of the linewidths taken from 11 measurements under the pressures at $294 \mathrm{~K}, \Delta B_{\|}=6.8(5) \mathrm{mT}, \Delta B_{\perp}=6.2(9) \mathrm{mT}$, are different and do not depend on the pressure values. The spectra were recorded up to $550 \mathrm{MPa}$ and then the pressure was gradually decreased. The reverse transition from LS to HS state occurs at about $380 \mathrm{MPa}$. Thus, this transition is accompanied by a large hysteresis effect.

The complicated character of the spin-crossover phenomenon under pressure is visible at temperatures below $270 \mathrm{~K}$. For example, as results from the measurements at $260 \mathrm{~K}$ (the evolution of the spectrum is shown in Fig. 3c), the pressure increase causes first, under $\sim 275 \mathrm{MPa}$, a transition from the HS to LS phase with $g$-values similar to the ones obtained under atmospheric pressure (Fig. 6a). Simultaneously, $\Delta B_{\perp}$ increases with pressure from $\sim 11 \mathrm{mT}$ at $275 \mathrm{MPa}$ to $\Delta B_{\perp}=13-14 \mathrm{mT}$ at $\sim 290 \mathrm{MPa}$, and is somewhat higher than at atmospheric pressure (see Fig. 6b). Then, at about $410 \mathrm{MPa}$, the shape of the LS spectrum changes again and it turns into a spectrum similar to that at room temperature under high pressure. So, there are two low-spin phases: the low-pressure LS1 below $410 \mathrm{MPa}$ and the high-pressure LS2, characterised by significantly different values of $g_{\perp}$ and $\Delta B_{\perp}$ as seen in Fig. 6 .

The transition to the LS2 phase can be induced only by pressure. At temperatures lower than $275 \mathrm{~K}$ a gradual transformation LS1 $\leftrightarrow$ LS2 can be observed. This transition exhibits a large hysteresis effect of about 100-110 MPa. Figure 7 shows that the LS2 phase replaces LS1 one suggesting that the seed domain of the pressure induced LS2 phase appears in the LS1 phase. At higher temperatures this process is much more rapid (for example, at $T=260 \mathrm{~K}$ (Fig. 6) transformation occurs at a pressure changing of about $10 \mathrm{MPa}$ instead of about $40-50 \mathrm{MPa}$ at $T=215 \mathrm{~K}$ (Fig. 7)). The number of the pressure induced LS2 complexes can significantly exceed that of the complexes in the LS1 phase. This is distinctly seen from the experiments at high temperatures. 

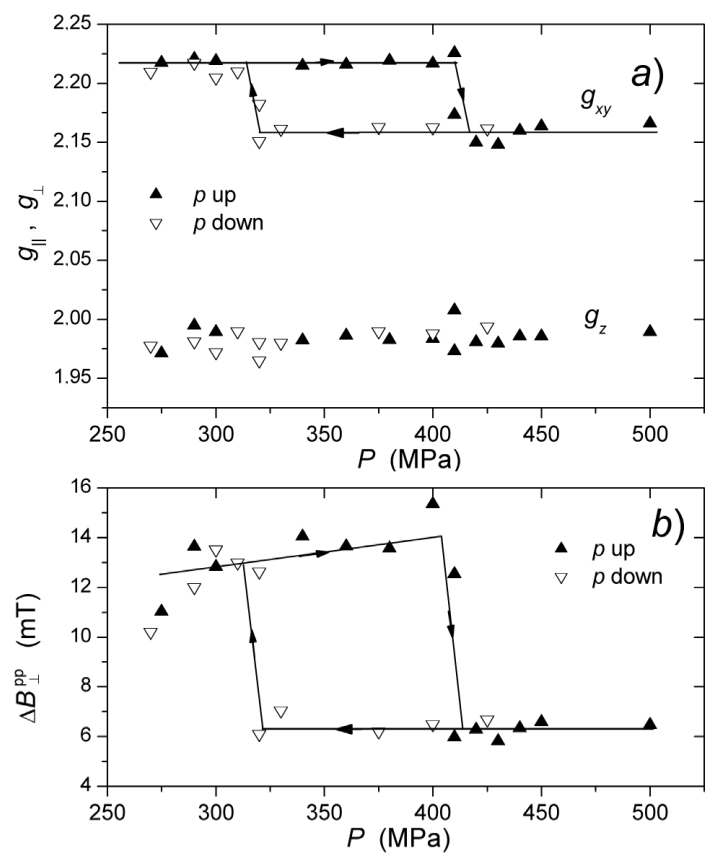

Fig. 6. The pressure dependence of $g_{\perp}=\left(g_{x}+g_{y}\right) / 2, g_{\|}=g_{z}$ components (a) and of $\Delta B_{\perp}^{\text {pp }}$ (a pick to pick linewidth) at $260 \mathrm{~K}$; (b) obtained from the fitting procedure.

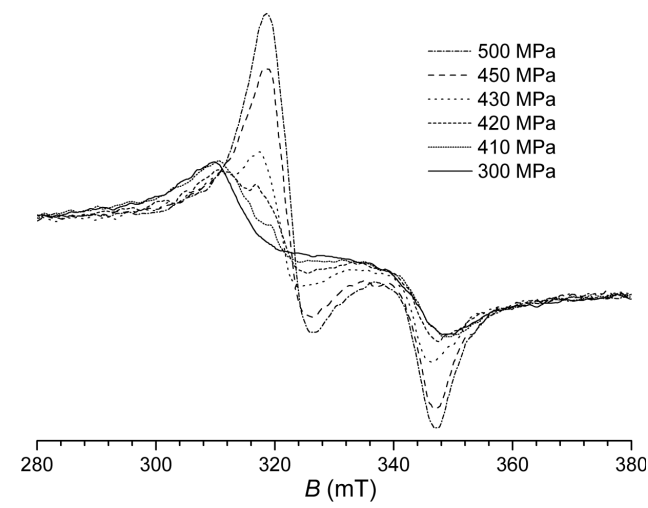

Fig. 7. The transformation of the EPR signals in the pressure induced transition at $T=215 \mathrm{~K}$.

Hydrostatic pressure also influences the HS $\leftrightarrow$ LS1 transition. Under atmospheric pressure in the temperature-induced transition, about $20 \%$ of the HS complexes undergo the transformation to the LS1 state in the interval 225-215 K (see Fig. 4). Under the hydrostatic pressure of $300 \mathrm{MPa}$ the transition temperature is shifted to about $260 \mathrm{~K}$, but the number of the temperature induced LS1 complexes decreases (in the interval of $\sim 10 \mathrm{~K}$ of their rapid increase) to about 
$10 \%$. Then, with decreasing temperature the content of the LS1 complexes reaches that observed in the experiment under the ambient pressure. Generally, the higher the temperature the higher the pressure needed for the HS $\leftrightarrow$ LS1 transition and the lower the amount of the LS1 complexes. It seems that the maximal number of the LS1 centres is determined mainly by temperature, and the pressure influences only the transition temperature. Hydrostatic pressure applied at temperatures of the LS1 complexes existence very slightly increases their number.

\subsection{Spin transition diagram}

The transition from the HS to LS phase in the (2Me-5Et-PyH) $\left[\mathrm{Fe}(\mathrm{Th}-5 \mathrm{Cl}-\mathrm{Sa})_{2}\right]$ is not complete. So, it is convenient to accept the characteristic temperature or pressure, $T_{a}$ or $P_{a}$, at which the LS signal appears or disappears in the EPR spectrum to describe a given phase transition. We used the values of $T_{a}$ and $P_{a}$ obtained at various temperatures and pressures to draw the following phase diagram (Fig. 8).

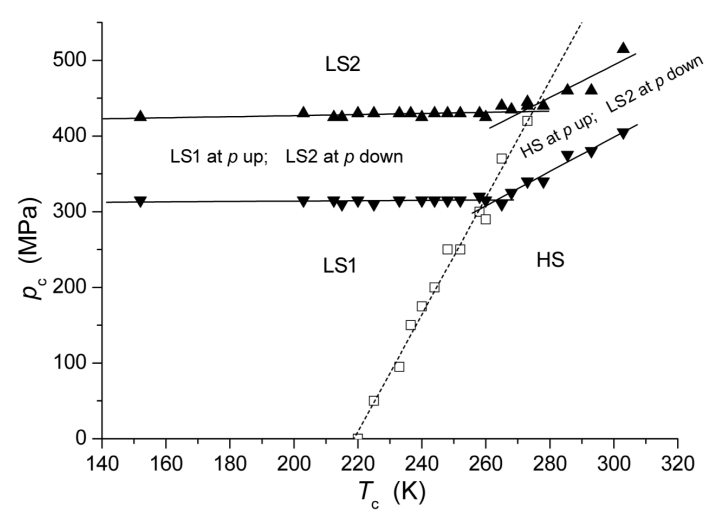

Fig. 8. The phase diagram for $(2 \mathrm{Me}-5 \mathrm{Et}-\mathrm{PyH})\left[\mathrm{Fe}(\mathrm{Th}-5 \mathrm{Cl}-\mathrm{Sa})_{2}\right]$ compound.

When temperature increases, the pressure of the spin transition HS $\leftrightarrow$ LS1 also increases with a slope coefficient $\mathrm{d} P_{a} / \mathrm{d} T_{a}=(7.7+0.3) \mathrm{MPa} / \mathrm{K}$, obtained from the linear dependence. In the LS1 phase, the values of $g_{\|}=1.988$ and $g_{\perp}=2.208$ are independent of temperature and pressure. Above $410 \mathrm{MPa}$, a new low-spin phase LS2 appears with different $g$-factors $\left(g_{\|}=1.997, g_{\perp}=2.173\right)$. The transition pressure is almost independent of temperature up to $275 \mathrm{~K}$ and equal $P=420 \mathrm{MPa}$. This is a triple point, at which all phases coexist. Above that point, the transition from the HS phase to LS2 phase is observed with a visible dependence on temperature, $(2.6 \pm 0.3) \mathrm{MPa} / \mathrm{K}$. A large hysteresis $(110 \mathrm{MPa}$ for both LS2 $\leftrightarrow$ LS1 and LS2 $\leftrightarrow$ HS transitions) is observed. This bistability range of temperatures and pressures, where the system can exist in two phases: HS or $\mathrm{LS}$, is the one of the most interesting characteristics of the phase diagram. 


\section{Discussion}

We shall discuss the observed spin crossover in (2Me-5Et-PyH) $\left[\mathrm{Fe}(\mathrm{Th}-5 \mathrm{Cl}-\mathrm{Sa})_{2}\right]$ in the framework of the approach developed in $[15,16,23]$, and based on the conception that the process of the spin transition is carried out through the arise and development of the domains of molecules in a new spin state (LS) in the matrix of molecules in the initial spin state (HS). EPR allows monitoring this process since the parameters of the EPR signal, its width and shape first of all, depend on the arrangement of paramagnetic complexes and interaction between them. The mere manifestation of exchange interactions between LS complexes, even at their very small formal concentrations in the crystal, have led to the conclusion that LS complexes are not distributed randomly in the structure of the compound but concentrate in small areas and permitted direct observation of domain formation. Changes in the exchange interaction between Fe(III) ions, which become apparent in the EPR linewidth, allowed characterisation of the domains and their behaviour. The mentioned conclusions were first made for the $\mathrm{Na}\left[\mathrm{Fe}(\mathrm{Th}-\mathrm{Sa})_{2}\right]$ compound showing a very gradual spin transition [16]. A subsequent study has shown that the process of domains formation can vary in different compounds. However, the main features of the response of the low-spin complexes arrangement in domain to the change of temperature or pressure remain unchanged [23].

In the spectra of $(2 \mathrm{Me}-5 \mathrm{Et}-\mathrm{PyH})\left[\mathrm{Fe}(\mathrm{Th}-5 \mathrm{Cl}-\mathrm{Sa})_{2}\right]$ the $\mathrm{LS} 1$ signal is visible from $220 \mathrm{~K}$. An estimation of its integral intensity shows that it corresponds to less than $1 \%$ of magnetic complexes. The width of the individual EPR line found in the fitting procedure is relatively small and shows a remarkable anisotropy (see Fig. 4). Therefore, we can conclude that in this case, just as in the previously studied ones, the LS complexes are gathered in the "spin domains" and their EPR signals are narrowed by the exchange interaction. The exchange interaction averages the corresponding $g$-tensor components and EPR signals of LS complexes when $\nu_{\text {ex }}(\mathrm{LS})>\Delta g_{i(a b)} \beta B$. Consequently a definite contribution to the observed linewidth appears [6]. Both, $g$-tensor and the linewidth, are anisotropic. The exchange-averaged signals are characterised by crystal $g$-values, which differ from the molecular $g$-values of the isolated complex.

Although we do not know the crystal structure of (2Me-5Et-PyH) $\left[\mathrm{Fe}(\mathrm{Th}-5 \mathrm{Cl}-\mathrm{Sa})_{2}\right]$, we can make some estimations of the exchange interactions between LS complexes. Comparison of typical geometries of the HS and LS coordinating $\mathrm{FeS}_{2} \mathrm{O}_{2} \mathrm{~N}_{2}$ polyhedrons in $\mathrm{Fe}$ (III) thiosemicarbazonates [3, 20] (containing 4 complexes in the unit cell) shows that the angles of misalignment for the two sets of complexes are of about $20^{\circ}$. This allows a supposition that the differences between the crystal and molecular $g$-values are not large, which means that the main contribution to the linewidth is determined by the dipole-dipole interactions. Exchange interactions modulate them leading to a narrowing of the EPR line [24]. The EPR signal broadened by the dipole-dipole interaction should have a Gaus- 
sian shape, while the experimental lines are Lorentzian. The estimation of the dipole-dipole interactions in the cubic lattice approach gives [24] the frequency of the electron exchange $\nu_{\mathrm{ex}}(\mathrm{LS})$

$$
\nu_{\mathrm{ex}}=\frac{20}{3} \frac{M_{2}}{\Delta \nu} \sim 1 \times 10^{9} \mathrm{sec}^{-1} .
$$

Here $\Delta \nu$ is the observed linewidth and $M_{2}=(3 / 5) g^{4} \beta^{4} h^{2} S(S+1) 8.5 d^{-6}$ is the second moment of the EPR line. $M_{2}$ is obtained in the point charge approach for the middle distance between magnetic ions $d \approx 9 \AA$ A. Taking into account delocalisation of unpaired electrons and a contribution to the observed linewidth due to the misalignment effect, $\nu_{\mathrm{ex}}(\mathrm{LS})$ will increase.

Under atmospheric pressure and at room temperature only the EPR signal of HS complexes is observed. The fact that EPR of Fe(III) complexes having $S=5 / 2$ is a single relatively broad $(\Delta B=81.5 \mathrm{mT})$ line, can be a result of either very small zero field splitting of $S=5 / 2$ state or of the presence of the strong enough exchange interactions averaging the fine structure and dipole-dipole interactions between $\mathrm{Fe}(\mathrm{III})$ ions. Both possibilities are supposed to be valid.

The temperature dependence of the LS complexes linewidth is unusual. In the previously studied compounds [16] we have observed a narrowing of the EPR signal corresponding to the growing number of the LS complexes. In the spectra of $(2 \mathrm{Me}-5 \mathrm{Et}-\mathrm{PyH})\left[\mathrm{Fe}(\mathrm{Th}-5 \mathrm{Cl}-\mathrm{Sa})_{2}\right]$ the initially observed signal is narrow. However, the spin transition, manifested as a rapid increase in the number of LS complexes, is accompanied by an increase in the linewidth of the EPR signal $\Delta B_{\perp, \|}$ at $225-210 \mathrm{~K}$. Then, below $213 \mathrm{~K}$ in the temperature range, in which a gradual increase in the number of the LS complexes takes place, the signal narrowing is observed. This unusual temperature dependence of the linewidth, in our opinion, can be a result of the interplay of exchange interactions between the complexes of different types. In the situation when the exchange-coupled complexes occupy neighbouring positions in the crystal lattice the parameters of HS-HS and HS-LS (with $S=1 / 2$ ) exchange interactions are known to be smaller than those of LS-LS interactions [16]. In the process of the domain development the mean distances between the LS complexes can change. This means that, under some conditions, the LS-HS and LS-LS exchange interactions can become comparable. We suppose that the nuclei of a new LS phase appear in the vicinity of some structural defects in the form of small but dense agglomerates, in which LS-LS exchange interactions exceed the LS-HS interactions. An appearance of LS agglomerates induces the spin transition resulting in a rapid increase in the number of LS complexes. Consequently, the arrangement of the LS and HS Fe complexes becomes more regular and the average density of LS complexes in the domains diminishes. This process results in a weakening of LS-LS exchange interactions. Additionally, the induced LS complexes are partly surrounded by the HS ones and interact with them. Such an interaction between non-equivalent magnetic centres can lead to a broadening of the EPR line [6]. Simultaneously, a decrease in the domain density 
slows down the spin transition. Then, with increasing density of LS complexes in the domains at $T<210 \mathrm{~K}$, (LS-LS) exchange will be stronger than (LS-HS) one, which means that the (LS-LS) exchange will determine the observed linewidth. As a result, the linewidth of the EPR signal decreases. In any case, let us point out once more that the observed $\Delta B_{\perp, \|}(T)$ dependence could not be explained on the supposition of a random LS complexes distribution, even in the case when LS complexes content is extremely small.

Figure 4 shows an increasing relative content of the LS phase with decreasing temperature, which approaches $40-50 \%$ at $T \rightarrow T_{\mathrm{c}} \approx 95-100 \mathrm{~K}$. We can consider that this process is influenced by interactions between different elements of the structure - interactions between LS complexes in domains of different densities as well as between LS complex and surroundings HS complexes (maybe it is more reasonably to speak about interactions between domains [14]). Determination of corresponding parameters of interactions is obviously an interesting but not a simple problem. We shall try to estimate the total interactions parameter $\Gamma$ on the basis of its relation to the number of LS molecules in the domain $n$ obtained by Gütlich et al. [25]

$$
\Gamma=2 R T_{\mathrm{c}}\left(1-\frac{1}{n}\right) .
$$

Here $R$ is the gas constant. This expression is derived on the basis of the thermodynamics of the spin transition for 1 mole of iron complexes. In (1) the temperature of the transition $T_{\mathrm{c}}$ corresponds to the same contents of the HS and LS complexes. We assume the validity of the thermodynamical consideration for the system including the LS domain and its nearest surroundings - some spheres of HS complexes (instead of $T_{\mathrm{c}}$ we shall use the corrected $T_{n}$ value corresponding to the final stage of LS density change in the domain). The number $n$ is determined by the balance between the energy gain due to stronger interactions of the LS molecules in a domain and the loss equivalent to the energy increase caused by disturbance of the HS crystal lattice by the LS complexes. In other words it is ruled by the condition of the energy minima in a given region of the crystal lattice. The temperature behaviour of $\Delta B_{\perp, \|}$, shown in Fig. 4, and the lack of the temperature dependence of $\Delta B_{\perp, \|}$ below $160 \mathrm{~K}$ allows a conclusion that the process of a dense domain formations comes to an end at $T=T_{n}=160 \mathrm{~K}$. The EPR signal of the LS complexes has the Lorentzian shape. This means that the intra-domain magnetic dipole-dipole interactions, differences in the Zeeman energies of the two sets of complexes and interactions of the boundary LS complexes with the HS neighbours are fully averaged by the exchange interactions between LS complexes. As far as the number of LS complexes in the domain $n$ is concerned, we can only conclude that it should be large enough to remove the features inherent to large clusters. The magnetic spectrum of a cluster is known to consist of a large number of levels corresponding to the possible values of the total spin $S$ and separated by the intervals comparable with the exchange parameter $J$. An increase in the num- 
ber $n$ leads to an increase in the numbers of the levels, a decrease in the intervals between the levels and to the loss of their individuality. Probably, the number $n$ of about several tens would be high enough, however, it is not necessary to try and estimate this number exactly. The expression (1) shows that for large $n$ the desired parameter $\Gamma$ has the order of magnitude of $2 R T_{n}$.

Some features of the HS $\leftrightarrow$ LS2 transition under hydrostatic pressure investigated in $(2 \mathrm{Me}-5 \mathrm{Et}-\mathrm{PyH})\left[\mathrm{Fe}(\mathrm{Th}-5 \mathrm{Cl}-\mathrm{Sa})_{2}\right]$, crossover effect itself, incompleteness of transition, and varying density of the LS complexes in the domains of a new phase are of similar character to those observed and discussed previously in some other Fe(III) thiosemicabazonates [16, 17, 23]. Let us consider the newly discovered phenomena: the existence of the additional pressure induced metastable LS phase and temperature independence of the pressure inducing the LS1 $\leftrightarrow$ LS2 transition. We have seen that a transition to the new LS phase (LS2), characterised by different $g_{i}$ and $\Delta B_{i}$ parameters of the EPR spectrum of LS complexes, happens even at room temperature (at $P=460 \mathrm{MPa}$ ). A temperature decrease $\sim 275 \mathrm{~K}$ causes a reduction in $P_{a}$ of the transition to the value of $420 \mathrm{MPa}$ and below that temperature the transition to LS2 occurs through LS1, in which the parameters of the LS complexes, even at pressures close to the critical one, are the same as those of the LS-phase induced by decreasing temperature. In low temperatures the LS1 $\leftrightarrow$ LS2 spin transition takes place at the same pressure, of about $420 \mathrm{MPa}$. The change in the EPR parameters at this transition and large $P$-hysteresis (of $\sim 110 \mathrm{MPa}$ ) of the inverse LS2 $\leftrightarrow$ LS1 transition indicates the occurrence of the pressure induced structural phase transition. We relate this phase transition to a definite reorganization of the system of intermolecular contacts (of the H-bonds type) in the crystal structure of the compound, which results in a certain conformation of the $\mathrm{Fe}(\mathrm{III})$-carbazonate molecule. Of course, $g_{i}$ and $\Delta B_{i}$ parameters remain crystal ones. The change in the parameters observed at the phase transition is a consequence of changes in the molecular parameters and not in the exchange-averaged crystal values. In the latter case the trace of $\{g\}$-tensors should be constant but it is not the case.

The fact that the phase transition arises at the same pressure and does not depend on temperature (below the triple point) implies that some barrier of the free energy $G$ exists and should be overcome to change the specific volume by $\Delta V=\partial G_{1} / \partial P-\partial G_{2} / \partial P$ and to reorganize the system of contacts. It is not enough to decrease temperature to overcome this barrier and only an increase in the hydrostatic pressure permits it. The specificity of the phase transition in question is that it occurs not in the bulk of the initial HS phase but in the nucleating centres of LS1 phase. As one can see from Fig. 3c and Fig. 7, at pressures higher than $410 \mathrm{MPa}$ the LS2 centres arise in the LS1 domain as the nuclei of a new phase. Only above the triple point the nuclei of LS2 phase appear in the HS phase. The $T_{a}$ pressure, necessary for inducing this process is higher than at low temperatures and increases with temperature. It should be mentioned that 
the EPR parameters of LS2 centres, linewidth first of all, which is even smaller than the EPR linewidth of LS1 complexes, do not change after LS2 signal appearance. This means that the LS2 nuclei include from the very beginning a sufficiently great number of complexes and that the number of LS1 complexes in the domain is large indeed. At temperatures close to, but lower than the triple point the LS2 phase is supposed to appear and develop together with the LS1 phase. The small volume of the LS1 phase does not allow accurate monitoring of this process. It is possible to conclude that LS2 is observed from the very beginning in a form of quite dense agglomerates, which developed faster than those of LS1. It should be emphasised that the rate of the LS2 phase development depends on temperature. Figure 3 also shows that at $T=260 \mathrm{~K}$ the LS1 $\rightarrow$ LS2 transition happens very quickly: it arises at pressure $P=410 \mathrm{MPa}$ and already at $P=420 \mathrm{MPa}$ LS2 complexes completely replace LS1 ones and the subsequent growth of the LS2 phase occurs directly from the HS phase as it takes place in the HS-LS2 transition at $T=294 \mathrm{~K}$. However, at $T=215 \mathrm{~K}$ this transition demands that $\Delta P \sim 40 \mathrm{MPa}$ (Fig. 7), probably due to a smaller compressibility of the crystal at low temperatures. The LS1 $\leftrightarrow$ LS2 transition possesses all features of the first order phase transition, including large enough hysteresis, though the relative content of LS2 phase is small: for example, for $T=240 \mathrm{~K}$ and $P=500 \mathrm{MPa}$ it is not greater than $\sim 16 \%$. We consider these circumstances as a serious argument for the possibility to describe the state of the complexes in the domains and their surroundings at the thermodynamical equilibrium in the usual way using the main thermodynamic parameters.

\section{Conclusions}

A new feature of the HS $\leftrightarrow$ LS transition, that is the transition of the LS phase into a new state, was discovered in $(2 \mathrm{Me}-5 \mathrm{Et}-\mathrm{PyH})\left[\mathrm{Fe}(\mathrm{Th}-5 \mathrm{Cl}-\mathrm{Sa})_{2}\right]$. This transition was shown to be induced only by the hydrostatic pressure exceeding some critical value and did not depend on temperature below that corresponding to the triple point. This spin transition was characterised by all features of the first order phase transition and showed large $P$-dependent hysteresis of about 110 MPa. Hence, a new type of bistability was found. New features of the character and process of the HS $\leftrightarrow$ LS1 spin transitions were demonstrated assuming the concept of the domain appearance and development.

\section{References}

[1] P. Gütlich, H.A. Goodwin, in: Spin Crossover in Transition Metal Compounds I, Eds. P. Gütlich, H.A. Goodwin, in series Topics in Current Chemistry, Vol. 233, Springer Verlag, Heidelberg 2004, p. 1.

[2] P. Gütlich, A. Hauser, H. Spiering, Angew. Chem. Int. Ed. Engl. 33, 2024 (1994).

[3] V.A. Kogan, V.V. Zelentsov, G.M. Larin, V.V. Lukov, Transition Metal Complexes with Hydrazones, Nauka, Moscow 1990, p. 85 (in Russian). 
[4] P.J. van Koningsbruggen, Y. Maeda, H. Oshio, in: Spin Crossover in Transition Metal Compounds I, Eds. P. Gütlich, H.A. Goodwin, in series Topics in Current Chemistry, Vol. 233, Springer Verlag, Heidelberg 2004, p. 259.

[5] S. Floquet, M.-L. Boillot, E. Riviére, F. Varret, K. Boukheddaden, D. Morineau, Ph. Négrier, New J. Chem. 27, 341 (2003).

[6] A. Abragam, B. Bleaney, Electron Paramagnetic Resonance of Transition Ions, Clarendon Press, Oxford 1970.

[7] B.J. Kennedy, A.C. McGrath, K.S. Murray, B.W. Skelton, A.H. White, Inorg. Chem. 26, 483 (1987).

[8] Y. Maeda, N. Tsutsumi, Y. Takashima, Inorg. Chem. 23, 2440 (1984).

[9] M. Mohan, N.S. Gupta, L. Chandra, N.K. Jha, R.S. Prasad, Inorg. Chim Acta 141, 185 (1988).

[10] M.D. Timken, C.E. Strouse, S.M. Soltis, S.A. Daverio, D.N. Hendrikson, A.M. Abbel-Mawgoud, S.R. Wilson, J. Am. Chem. Soc. 108, 395 (1986).

[11] A.J. Conti, R.K. Chadha, K.M. Sena, A.L. Rheingold, D.N. Hendrickson, Inorg. Chem. 32, 2670 (1993).

[12] M.S. Haddad, W.D. Federer, M.W. Lynch, D.N. Handrickson, Inorg. Chem. 20, 131 (1981).

[13] M.M. Bhadbhade, D. Srinivas, Polyhedron 17, 2699 (1998).

[14] C. Cantin, J. Kliava, A. Marbeuf, D. Mikhailitchenko, Eur. Phys. J. B 12, 415 (1999).

[15] Yu.V. Yablokov, V.V. Zelentsov, M. Augustyniak-Jabłokow, A. Krupska, J. Mroziński, Matter. Sci. 21, 215 (2003).

[16] V.V. Zeletsov, Yu.V. Yablokov, M.A. Augustyniak-Jabłokow, A. Krupska, J. Mroziński, V.A. Ulanov, Chem. Phys. 301, 15 (2004).

[17] P. Paganiuch, S. Decurtins, P. Gütlich, J. Am. Chem. Soc. 112, 3270 (1990).

[18] P. Gütlich, A.B. Gaspar, V. Ksenofontov, Y. Garcia, J. Phys., Condens. Matter 16, S1087 (2004).

[19] P. Gütlich, V. Ksenofontov, A.B. Gaspar, Coord. Chem. Rev. 249, 1811 (2005).

[20] N.A. Ryabova, V.I. Ponomarev, V.V. Zelentsov, L.O. Atovmyan, Sov. Phys. Crystallogr. (engl. transl.) 26, 53 (1981); ibid. 27, 46 (1982); ibid. 27, 171 (1982); N.A. Ryabova, V.I. Ponomarev, L.O. Atovmyan, V.V. Zelentsov, V.I. Shipilov, Sov. J. Coord. Chem. (Transl. of Zh. Koord. Khimii) 4, 95 (1978).

[21] V.V. Zelentsov, A.V. Ablov, K.I. Turta, R.A. Stukan, N.N. Gerbeleu, E.V. Ivanov, A.P. Bogdanov, N.A. Barba, V.G. Bodyu, Russ. J. Inorg. Chem (Transl. of Zh. Neorg. Khim) 17, 1000 (1972).

[22] M. Krupski, Rev. Sci. Instrum. 67, 2894 (1996).

[23] Yu.V. Yablokov, V.V. Zelentsov, M.A. Augustyniak-Jablokow, A. Krupska, J. Mrozinski, in: Modern Problems in Condensed Matter Physics, Eds. B.Z. Malkin, Yu.N. Proshin, CJC "Novoe znanie", Kazan 2004, p. 367.

[24] S.A. Altshuler, B.M. Kozyrev, Electron Paramagnetic Resonance in Compounds of Transition Elements, 2nd ed., Wiley, New York 1974, ch. IV.

[25] P. Gütlich, H. Köppen, R. Link, H.G. Steinhäuser, J. Chem. Phys. 70, 3977 (1979). 